\title{
COMPARATIVE ANALYSIS OF DIFFERENT CAD METHODS FOR EXTRACTION OF THE HEMT NOISE WAVE MODEL PARAMETERS
}

UDC (004.032.26+621.3.049.77):613.644

\section{Vladica Đorđević, Zlatica Marinković, Olivera Pronić-Rančić}

University of Niš, Faculty of Electronic Engineering, Department of Telecommunications, Niš, Republic of Serbia

\begin{abstract}
The noise wave model has appeared as a very appropriate model for the purpose of transistor noise modeling at microwave frequencies. The transistor noise wave model parameters are usually extracted from the measured transistor noise parameters by using time-consuming optimization procedures in microwave circuit simulators. Therefore, three different Computer-Aided Design methods that enable more efficient automatic determination of these parameters in the case of high electron-mobility transistors were developed. All of these extraction methods are based on different noise de-embedding procedures, which are described in detail within this paper. In order to validate the presented extraction methods, they were applied for the noise modeling of a specific GaAs high electron-mobility transistor. Finally, the obtained results were used for the comparative analysis of the presented extraction approaches in terms of accuracy, complexity and effectiveness.
\end{abstract}

Key words: artificial neural networks, computer-aided design, GaAs HEMT, noise parameters, noise wave model

\section{INTRODUCTION}

The transistor noise characterization is usually done by using the following noise parameters: $F_{\text {min }}$ - minimum noise figure, $\Gamma_{o p t}$ - optimum source reflection coefficient and $r_{n}$ normalized noise resistance. Nowadays, the measurements of these parameters are performed effectively using modern measurement equipment that is very expensive and often unavailable to the circuit designers. For this reason, a large number of circuit designers still uses the older and cheaper measurement setups, resulting in time-consuming noise measurements. Consequently, different transistor noise models were developed [1-9] in

Received January 17, 2017

Corresponding author: Vladica Đorđević

University of Niš, Faculty of Electronic Engineering, Aleksandra Medvedeva 14, 18000 Niš, Republic of Serbia

E-mail: vladica.djordjevic@elfak.rs 
order to provide more efficient transistor noise analysis within circuit simulators. Most of the proposed transistor noise models represent the noise generated in a component using equivalent voltage and/or current sources [3, 5-6]. However, at microwave frequencies, a wave representation of noise looks attractive since it allows the use of scattering matrices for the noise computations, leading to advantages in Computer-Aided Design (CAD) of microwave networks [2, 7-18]. The power of the wave representation of noise lies in its ability to treat the network noise in terms of incident and reflected waves, which is compatible with the scattering matrix description of microwave networks. In this way, the whole noise analysis can be performed using scattering parameters. Therefore, the wave representation of noise provides a suitable alternative to the most commonly used representations of noise generated in network based on additional voltage and/or current noise sources.

The parameters of the noise wave model, called the noise wave temperatures, are extracted from the transistor measured noise parameters, usually using optimization procedures in microwave circuit simulators. As the noise wave temperatures are bias, temperature and frequency [13] dependent, it is necessary to perform the extraction of these parameters for each operating condition and frequency separately. Due to that, in situations when repeated extractions are needed, optimization procedures become timeconsuming, which is quite undesirable from the aspect of efficient transistor noise modeling. Hence, it would be useful to develop certain extraction procedures that would provide more efficient determination of these temperatures. Moreover, because in the case of noise wave model there are closed form expressions relating the noise wave temperatures and the noise parameters of transistor intrinsic circuit [2], the optimization procedures can be avoided by applying the de-embedding procedures to determine the intrinsic noise parameters from the measured transistor noise parameters [19-23], and further calculation of the noise wave temperatures.

In this paper, three different CAD methods for extraction of the noise wave temperatures in the case of GaAs high electron-mobility transistor (HEMT) are presented. This type of transistor is chosen because it has very good noise properties at microwave frequencies, so it is widely used in low-noise components in modern microwave communication systems. The presented methods provide de-embedding of the noise parameters of transistor intrinsic circuit from the measured transistor noise parameters and further straightforward extraction of the transistor noise wave temperatures. All of these extraction methods are based on different noise de-embedding procedures: analytical procedure, procedure within a microwave circuit simulator and procedure using artificial neural networks (ANNs) [19-23]. In order to determine which of these extraction methods is the most suitable for the transistor noise modeling, they are applied to a specific GaAs HEMT component, and the obtained results are used for the comparative analysis of the presented extraction procedures.

The article is organized as follows. After Introduction, a short description of the wave representation of transistor noise is given in Section 2. Three different CAD extraction methods are presented in Section 3. Section 4 contains the achieved modeling results and the comparative analysis of the presented extraction procedures. Finally, the most important conclusions are given in the last section. 


\section{WAVE-BASED REPRESENTATION OF NOISY TWO-PORT MICROWAVE NETWORKS}

The noise wave model of the GaAs HEMT device in a packed form is the subject of research presented in this paper. Equivalent circuit of packaged HEMT is shown in Fig. 1 [24]. It consists of intrinsic and extrinsic parts. The intrinsic circuit is framed with a dashed line, and intrinsic noise parameters are referred to it. The remainder of the equivalent circuit elements, embedded in the extrinsic circuit, represents parasitic effects and the package.

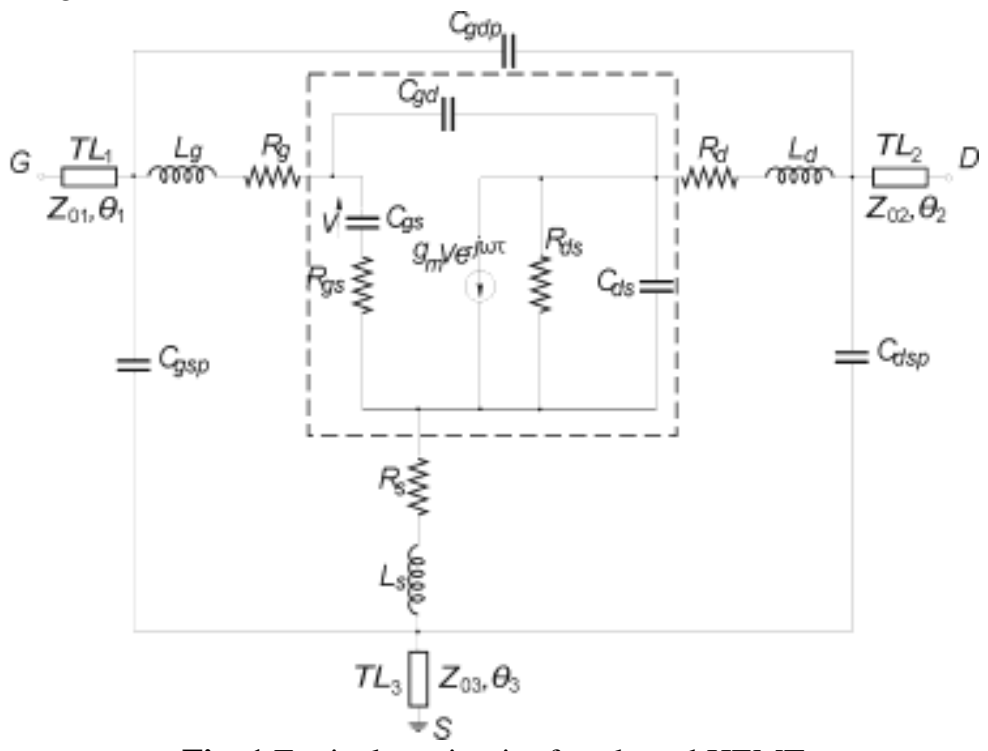

Fig. 1 Equivalent circuit of packaged HEMT

The transistor intrinsic circuit is a linear noisy two-port network. In the case of the noise wave representation, it can be considered as a noiseless two-port defined by transfer scattering parameters $[T]$ with additional noise wave sources, $a_{n}$ and $b_{n}$, referring to the input, Fig. 2 [2]. The matrix equation describing this noisy two-port representation is as follows:

$$
\left[\begin{array}{l}
a_{1} \\
b_{1}
\end{array}\right]=\left[\begin{array}{ll}
T_{11} & T_{12} \\
T_{21} & T_{22}
\end{array}\right]\left[\begin{array}{l}
b_{2} \\
a_{2}
\end{array}\right]+\left[\begin{array}{l}
a_{n} \\
b_{n}
\end{array}\right],
$$

where $a_{i}$ and $b_{i}, i=1,2$, are incident and output waves at the $i$-th port.

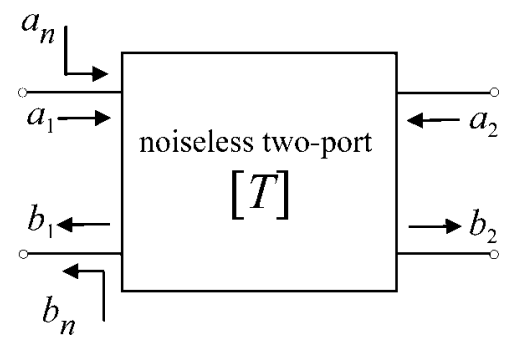

Fig. 2 Two port noisy network 
The elements of the noise source vector are correlated and characterized by correlation matrix $C_{T}$ :

$$
C_{T}=\left[\begin{array}{cc}
\left\langle\left|a_{n}\right|^{2}\right\rangle & \left\langle-a_{n} b_{n}^{*}\right\rangle \\
\left\langle-b_{n} a_{n}^{*}\right\rangle & \left\langle\left|b_{n}\right|^{2}\right\rangle
\end{array}\right],
$$

where \langle\rangle indicates time average of the quantity inside and $*$ indicates complex conjugation.

However, it is shown that it is the most useful to use the noise wave temperatures as the noise wave model parameters $[2,12]$. Therefore, the correlation matrix $C_{T}$ can be expressed by the noise wave temperatures - two real temperatures, $T_{a}$ and $T_{b}$, and one complex correlation temperature, $T_{c}$, as:

$$
C_{T}=k \Delta f\left[\begin{array}{cc}
T_{a} & \left|T_{c}\right| e^{j \varphi_{c}} \\
\left|T_{c}\right| e^{-j \varphi_{c}} & T_{b}
\end{array}\right],
$$

where $k$ is the Boltzmann's constant and $\Delta f$ is the noise bandwidth ( $\Delta f=1 \mathrm{~Hz}$ is assumed).

Further, the noise wave temperatures can be expressed in terms of the noise parameters of transistor intrinsic circuit - minimum noise figure, $F_{m i n, i}$, optimum source reflection coefficient, $\Gamma_{o p t, i}=\left|\Gamma_{o p t, i}\right| e^{j \varphi_{o p t, i}}$, and noise resistance, $R_{n, i}$, as follows [2]:

$$
\begin{gathered}
T_{a}=T_{0}\left(F_{\text {min }, i}-1\right)+\frac{4 R_{n, i} T_{0}\left|\Gamma_{o p t, i}\right|^{2}}{Z_{0}\left|1+\Gamma_{o p t, i}\right|^{2}} \\
T_{b}=\frac{4 R_{n, i} T_{0}}{Z_{0}\left|1+\Gamma_{\text {opt }, i}\right|^{2}}-T_{0}\left(F_{\text {min }, i}-1\right) \\
T_{c}=\frac{4 R_{n, i} T_{0} \Gamma_{o p t, i}}{Z_{0}\left|1+\Gamma_{o p t, i}\right|^{2}}
\end{gathered}
$$

where $Z_{0}$ is the normalization impedance $(50 \Omega)$ and $T_{0}$ is the standard reference temperature $(290 \mathrm{~K})$.

\section{CAD PROCEDURES FOR EXTRACTION OF THE NOISE WAVE TEMPERATURES}

As already mentioned, optimization procedures in microwave circuit simulators that are usually used for extraction of the transistor noise wave temperatures require a lot of time. Therefore, three different more efficient CAD extraction methods related to the equivalent circuit of HEMT in a packaged form shown in Fig. 1 are developed. All of these extraction methods are carried out in two steps. The first step is de-embedding of the transistor intrinsic noise parameters from the noise parameters of the whole device. For that purpose, each of three developed extraction methods uses different noise deembedding procedure [19-23], and all the used de-embedding procedures will be explained bellow. The second step is the same in all three cases and represents determination of the 
noise wave temperatures from the de-embedded intrinsic noise parameters using the Eqs. (4)-(6) [2]. Since the second step is straightforward, the comparative analysis of the proposed CAD extraction methods, which will be given in the next section, will mainly refer to the developed noise de-embedding procedures.

\subsection{Analytical noise de-embedding procedure}

Analytical noise de-embedding procedure [19-21] is similar to the analytical smallsignal de-embedding procedure and provides determination of the transistor intrinsic noise parameters based on the noise parameters of entire device by removing the influence of the extrinsic circuit elements to the transistor noise. Namely, the main concept is to eliminate the noise influence of the extrinsic elements connected in cascade, series and parallel using $A B C D, Z$ and $Y$ representations, respectively.

Since the main concept of analytical noise de-embedding procedure is based on the noise correlation matrix manipulations, first, it is necessary to determine $A B C D$ noise correlation matrix of entire transistor based on its measured noise parameters:

$$
C_{A}=2 k T_{0}\left[\begin{array}{cc}
R_{n} & \frac{F_{\text {min }-1}}{2}-R_{n} Y_{\text {opt }}{ }^{*} \\
\frac{F_{\text {min }-1}}{2}-R_{n} Y_{\text {opt }} & R_{n}\left|Y_{\text {opt }}\right|^{2}
\end{array}\right],
$$

where $Y_{\text {opt }}$ is optimum source admittance associated to $\Gamma_{o p t}$.

After that, to remove the influence of the extrinsic elements to the transistor noise, the following equations are used:

$$
C_{A, d}=A_{e}^{-1}\left(C_{A, e}-C_{A}\right)\left(A_{e}{ }^{H}\right)^{-1}
$$

for the elements connected in cascade,

$$
C_{Z, d}=C_{Z}-C_{Z, e}
$$

for the series elements and

$$
C_{Y, d}=C_{Y}-C_{Y, e}
$$

for the shunt elements. In Eqs. (8)-(10), $C_{A, d}, C_{Z, d}$ and $C_{Y, d}$ are resulting de-embedded noise correlation matrices, $C_{A}, C_{Z}$ and $C_{Y}$ are starting noise correlation matrices, $C_{A, e}, C_{Z, e}$ and $C_{Y, e}$ are noise correlation matrices of the circuit elements to be de-embedded, $A_{e}$ is $A B C D$ matrix of the circuit elements to be de-embedded, while $H$ indicates Hermitian complex conjugate transpose.

The noise correlation matrix of a passive network such as the series and shunt circuit elements embedded in the transistor extrinsic circuit can be determined with only thermal noise sources from its $Z$ and $Y$ parameters, respectively, and temperature:

$$
\begin{gathered}
C_{Z, e}=2 k T_{0} \operatorname{Re}\left(Z_{e}\right), \\
C_{Y, e}=2 k T_{0} \operatorname{Re}\left(Y_{e}\right),
\end{gathered}
$$


where $Z_{e}$ and $Y_{e}$ are $Z$ and $Y$ matrices of the series and shunt elements embedded in transistor extrinsic circuit, respectively.

Also, the analytical noise de-embedding procedure requires conversions between $Z, Y$ and $A B C D$ noise correlation matrices [25]. In particular, the conversion formulas for the noise correlation matrix are based on the following equation:

$$
C^{\prime}=T \times C \times T^{*},
$$

where $C$ and $C^{\prime}$ represent noise correlation matrices before and after conversion, respectively, and $T$ represents transformation matrix that depends only on the conventional electrical matrix of the network.

It should be noted that since the small-signal circuit element matrices are needed for the noise correlation matrix conversions, small-signal de-embedding procedure must precede the noise de-embedding procedure.

Finally, the obtained noise correlation matrix of transistor intrinsic circuit should take the $A B C D$ form in order to calculate the intrinsic noise parameters using the following equations $[19,21]$ :

$$
\begin{gathered}
F_{\text {min }, i}=1+\frac{1}{k T_{0}}\left(\operatorname{Re}\left(C_{A, i_{12}}\right)+\sqrt{C_{A, i_{11}} C_{A, i_{22}}-\left(\operatorname{Im}\left(C_{A, i_{12}}\right)\right)^{2}}\right), \\
Y_{o p t, i}=\frac{\sqrt{C_{A, i_{11}} C_{A, i_{22}}-\left(\operatorname{Im}\left(C_{A, i_{12}}\right)\right)^{2}}+j \operatorname{Im}\left(C_{A, i_{12}}\right)}{C_{A, i_{11}}}, \\
\Gamma_{o p t, i}=\frac{Y_{0}-Y_{o p t, i}}{Y_{0}+Y_{o p t, i}}, \\
R_{n, i}=\frac{C_{A, i_{11}}}{2 k T_{0}},
\end{gathered}
$$

where $C_{A, i}$ is $A B C D$ noise correlation matrix of the transistor intrinsic circuit and $Y_{o p t, i}$ is intrinsic optimum source admittance associated to $\Gamma_{o p t, i}$.

The complete analytical noise de-embedding procedure related to HEMT equivalent circuit shown in Fig. 1 is composed of four steps and presented in Fig. 3 [21]. Starting from the extrinsic plane, the two transmission lines at the gate and drain side $\left(T L_{1}\right.$ and $T L_{2}$ ) are de-embedded using the $A B C D$ formulation. The third transmission line at the source side $\left(T L_{3}\right)$ is treated as a short-circuited line, so it is de-embedded using the $Z$ formulation. The $Y$ formulation is used for de-embedding of all three capacitors $\left(C_{g s p}, C_{g d p}\right.$ and $\left.C_{d s p}\right)$. Finally, after de-embedding the last three inductors $\left(L_{g}, L_{d}\right.$ and $\left.L_{s}\right)$ and resistors $\left(R_{g}, R_{d}\right.$ and $\left.R_{s}\right)$ using the $Z$ formulation, the extrinsic noise contributors are completely deembedded, and noise parameters of transistor intrinsic circuit are obtained. 


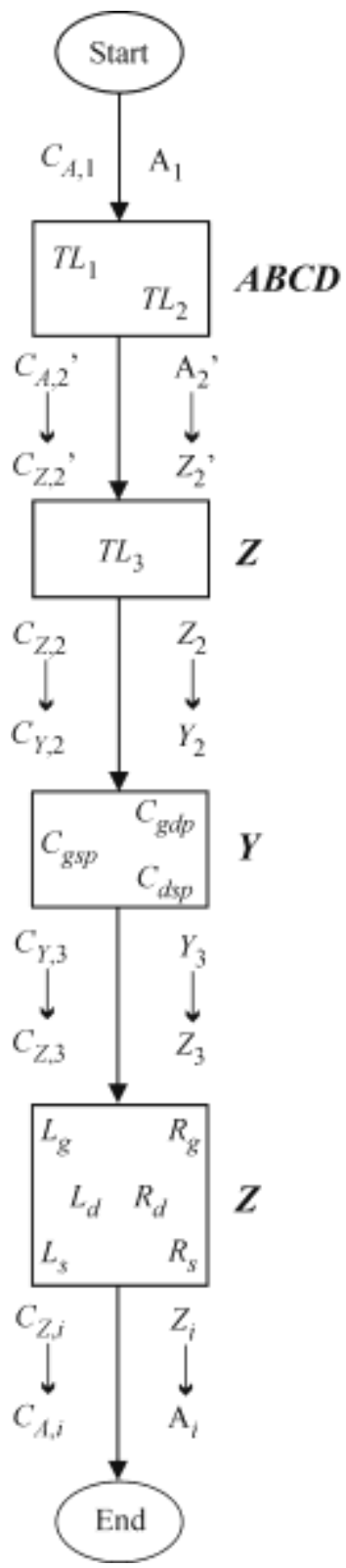

Fig. 3 Noise de-embedding procedure flowchart. The elements to be de-embedded are reported inside the boxes and the obtained matrices and their conversions are shown between the boxes. The formulation used $(A B C D, Z$ or $Y)$ is reported at the right side of the boxes 


\subsection{Noise de-embedding procedure within a microwave circuit simulator}

The starting point of this de-embedding procedure is to represent the transistor in a circuit simulator with its $S$ and noise parameters [22]. After that, the extrinsic equivalent circuit elements are added with the negative values and in the reverse order than in the case of transistor equivalent circuit shown in Fig. 1, with the aim to cancel their noise influence, Fig. 4 [22]. In this way, by simulating the resulting schematic, the noise parameters corresponding to the transistor intrinsic circuit are determined.

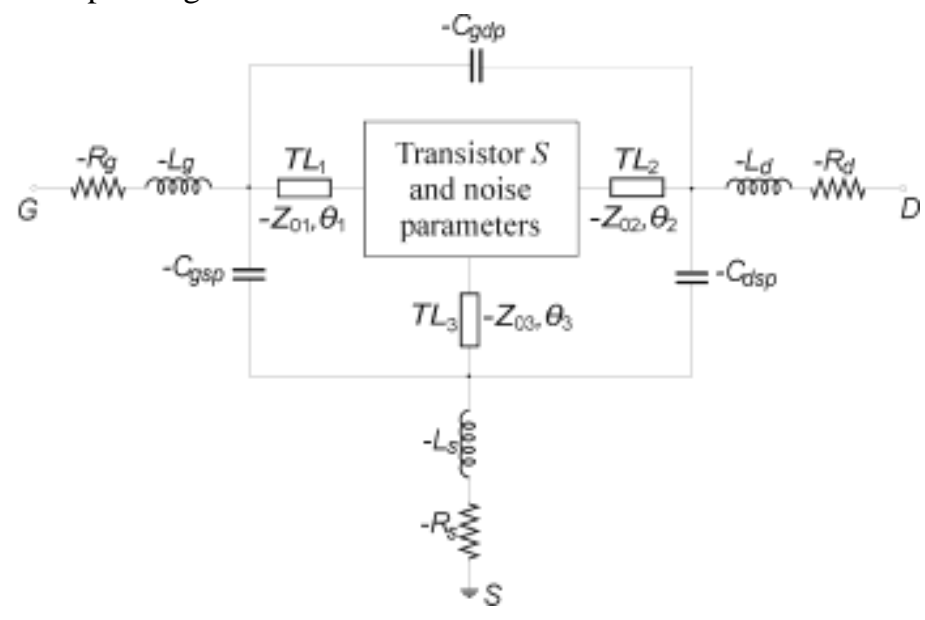

Fig. 4 The schematic for determination of intrinsic noise parameters of microwave HEMT within a microwave circuit simulator

\subsection{Noise de-embedding procedure using artificial neural networks}

The field of electrical engineering has proved to be a very suitable for the ANN applications [26-35]. By learning the dependence between two datasets, ANNs have the capability to approximate any nonlinear function, whereby the knowledge about the physical characteristics of the problem to be modeled is not needed [36]. Also, ANNs have a great capability to generalize, i.e. to determine response even for the input values that are not used for the model development. Moreover, since the working principle of ANNs is based on performing basic mathematical operations and calculating elementary mathematical functions, they give responses almost instantaneously [36].

Fig. 5 presents ANN approach proposed for the noise de-embedding of a transistor whose equivalent circuit is shown in Fig. 1 [23]. Namely, the presented ANN model is aimed at calculating the intrinsic noise parameters, $N_{i n t},\left(F_{m i n, i},\left|\Gamma_{o p t, i}\right|, \angle \Gamma_{o p t, i}\right.$ and $\left.r_{n . i}\right)$ from the transistor total noise parameters, $N_{\text {total }},\left(F_{\text {min }},\left|\Gamma_{\text {opt }}\right|, \angle \Gamma_{\text {opt }}\right.$ and $\left.r_{n}\right)$. The frequency, $f$, and ambient temperature, $T_{a m b}$, are also included as the ANN inputs, since the noise parameters are frequency and temperature dependent. Moreover, the equivalent circuit parameters, ECPs, are the additional ANN inputs. Therefore, the presented ANN has 25 neurons corresponding to the inputs and four output neurons corresponding to the $N_{\text {int }}$. 


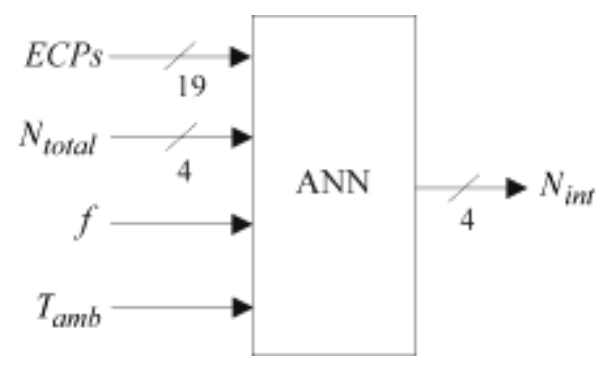

Fig. 5 The proposed ANN model for extraction of the intrinsic noise parameters of microwave HEMT

\section{NUMERICAL RESULTS AND DISCUSSION}

In order to compare the $\mathrm{CAD}$ methods for extraction of the noise wave temperatures based on different noise de-embedding procedures presented in the previous section, they were applied to a packaged HEMT, type NE20283A by NEC, working under different temperature conditions. The transistor measured $S$ and noise parameters were obtained in the frequency range $6-18 \mathrm{GHz}$ over the temperature range $233-333 \mathrm{~K}, 20 \mathrm{~K}$ step, by a measurement procedure described in [24]. The corresponding values of small-signal ECPs were taken from [24].

The analytical noise de-embedding procedure was developed by implementing the expressions given in Section 3.1 within MATLAB software environment [37].

The noise de-embedding procedure in a microwave circuit simulator was developed by implementing the schematic shown in Fig. 4 within ADS (Advanced Design System) circuit simulator [38].

In the case of the noise de-embedding procedure using ANNs, for the purpose of building an appropriate training set, the corresponding training values of $N_{\text {total }}$ and $N_{\text {int }}$ used for the ANN training were obtained by using the considered equivalent circuit of HEMT within ADS [39]. In order to properly cover input space, the values of ECPs that were used for the ANN training were samples obtained randomly in typical value ranges of ECPs within the frequency range 6-18 GHz and temperature range 233-333 K. To obtain ANN with the best performance, several ANNs based on Multi-Layer Perceptron (MLP) structure [36] with different number of hidden layers and hidden neurons were trained within MATLAB [37] using Quasi-Newton algorithm [36]. The network that gave the best results consists of two hidden layers, with nine neurons in the first and 12 neurons in the second layer. The training set used for its training contained 45000 samples.

The developed noise de-embedding procedures were used for extraction of the intrinsic noise parameters of the considered transistor, which were further used for determination of the noise wave temperatures. After that, the noise wave model was implemented within ADS [39] circuit simulator, the calculated noise wave temperatures were assigned to it, and the noise parameters of whole transistor were simulated in all three cases. The simulated noise parameters were compared with the measurements. 


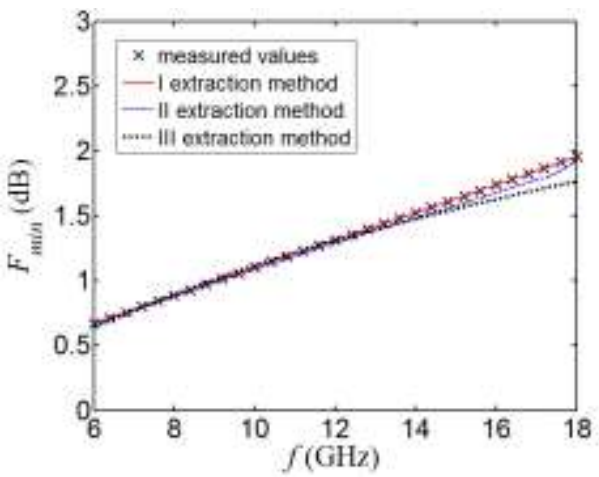

(a)

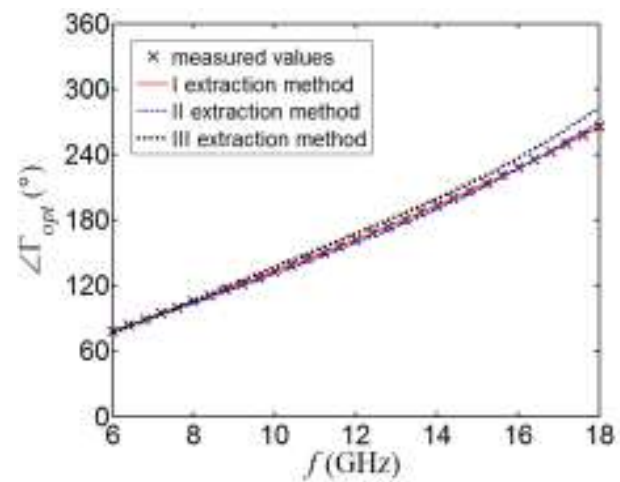

(c)

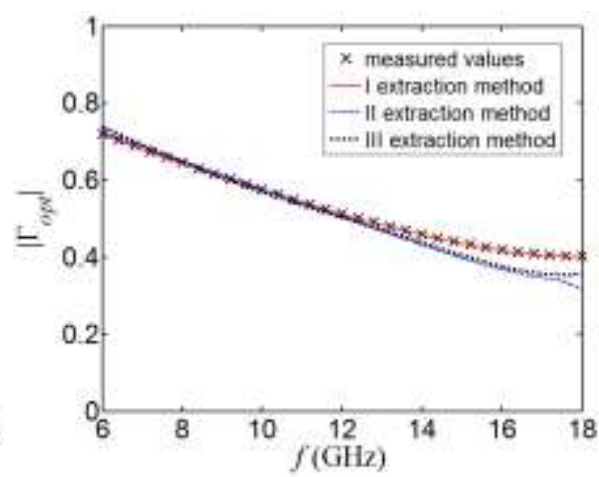

(b)

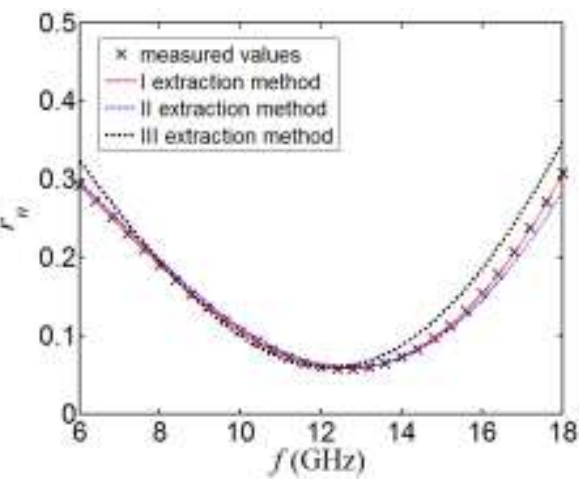

(d)

Fig. 6 Frequency dependence of the measured and simulated values of:
(a) $F_{\text {min }}$
(b) $\left|\Gamma_{\text {opt }}\right|$, (c)
(c) $\angle \Gamma_{o p t}$, and (d) $r_{n}$, at $293 \mathrm{~K}$

As an illustration, Fig. 6 presents the simulated $F_{\min },\left|\Gamma_{\text {opt }}\right|, \angle \Gamma_{\text {opt }}$ and $r_{n}$ in the case of the $\mathrm{CAD}$ extraction methods based on the analytical noise de-embedding procedure (I extraction method), noise de-embedding procedure within a microwave circuit simulator (II extraction method) and the noise de-embedding procedure using ANNs (III extraction method), and the corresponding measured data. The presented results were obtained for the ambient temperature of $293 \mathrm{~K}$, in the frequency range from 6 to $18 \mathrm{GHz}$.

Moreover, in order to express the accuracy of the noise modeling using proposed CAD extraction methods, average error $(A E)$ and maximum error $(M E)$, which are defined bellow, were calculated based on the results shown in Fig. 6 and presented in Table 1.

$A E$ is defined by using the mean value of the absolute value of the relative error $|\bar{\delta}|$ :

$$
A E=|\bar{\delta}|=\frac{1}{m} \sum_{j=1}^{m}\left|\delta_{j}\right|,
$$

where $m$ is the number of samples of datasets containing the parameters' simulated and target (measured) values, and $\delta_{j}$ is the relative error of the $j$-th sample. Namely, $\delta_{j}$ can be determined in the following way: 


$$
\delta_{j}=\frac{y_{j}-d_{j}}{d_{\max }-d_{\min }},
$$

where $y_{j}$ and $d_{j}$ are the $j$-th samples of datasets containing the parameters' simulated and target values, respectively, while $d_{\max }$ and $d_{\min }$ are the maximum and minimum parameters' target values, respectively.

$M E$ is defined as:

$$
M E=\max _{j=1}^{m}\left|\delta_{j}\right|
$$

Table 1 Test errors for the modeled noise parameters in the case of presented extraction methods

\begin{tabular}{ccccccc}
\hline & \multicolumn{2}{c}{ I extraction method } & \multicolumn{2}{c}{ II extraction method } & \multicolumn{2}{c}{ III extraction method } \\
\cline { 2 - 7 } & $A E(\%)$ & $M E(\%)$ & $A E(\%)$ & $M E(\%)$ & $A E(\%)$ & $M E(\%)$ \\
\hline$F_{\min }$ & 0.160 & 0.257 & 2.321 & 5.620 & 3.742 & 14.794 \\
$\left|\Gamma_{\text {opt }}\right|$ & 0.482 & 1.029 & 7.085 & 26.834 & 6.248 & 15.774 \\
$\angle \Gamma_{\text {opt }}$ & 0.055 & 0.091 & 1.154 & 2.049 & 3.099 & 9.240 \\
$r_{n}$ & 0.201 & 0.670 & 2.465 & 9.422 & 6.251 & 16.103 \\
\hline
\end{tabular}

As can be seen in Fig. 6 and Table 1, a good noise parameter modeling accuracy was achieved for all three presented extraction methods. However, I extraction method provides slightly better modeling results compared to the other two methods. On the other hand, since the noise de-embedding procedure applied in I extraction method is mainly based on matrix manipulations, it requires mathematically demanding calculations.

As the noise de-embedding procedure within a microwave circuit simulator is based on the implementation of the schematic whose extrinsic elements have the negative values and the reverse order than in the case of the considered transistor equivalent circuit, II extraction method is the simplest extraction method compared to the other two methods. Nevertheless, such a transistor noise de-embedding approach cannot be performed in all circuit simulators. This is because of the fact that some of the simulators do not support noise calculation of negative resistances [22]. In this case, the noise de-embedding procedure was performed in Agilent ADS 2002 [38]. However, the higher versions of ADS do not support the noise de-embedding. For this reason, before applying this noise de-embedding procedure, one should check on an appropriate example if the procedure can be performed in the considered simulator.

III extraction method has lower accuracy compared to I extraction method and greater computational complexity compared to II extraction method. However, it ensures avoiding of the problems related to the other two extraction methods. Namely, the noise de-embedding procedure using ANNs does not require developing complex matrix calculations as needed in the analytical noise de-embedding procedure. Moreover, the noise de-embedding procedure using ANNs does not require specific (older) versions of circuit simulator like in the case of the noise de-embedding procedure within a microwave circuit simulator. Therefore, III extraction method can be used as a suitable alternative to the other two methods. 


\section{CONCLUSION}

The noise wave temperatures are extracted from the measured transistor noise parameters usually by using optimization procedures within microwave circuit simulators. However, as the noise wave temperatures are bias, temperature and frequency dependent, the optimization procedures used for their extraction are time-consuming. In this paper, three efficient CAD extraction methods are presented. The presented extraction methods are carried out in two steps. The first step is de-embedding of the intrinsic noise parameters from the measured transistor noise parameters. For that purpose, each of the proposed extraction methods uses different noise de-embedding procedure: analytical noise de-embedding procedure, noise de-embedding procedure within a microwave circuit simulator and noise de-embedding procedure using ANNs. The second step is calculation of the noise wave temperatures based on the determined intrinsic noise parameters using the noise wave model expressions.

The presented CAD extraction methods were applied to a specific HEMT device in a packaged form. The corresponding noise parameters of entire transistor circuit were simulated in the circuit simulator using the obtained noise wave temperatures in all three cases. A good agreement between the simulated and measured transistor noise parameters was achieved for all three CAD extraction methods. However, each of the presented methods has advantages and limitations. Therefore, the final decision about the choice of the most appropriate CAD extraction method depends on the user preferences and availability of appropriate software environments.

Acknowledgement: The work was supported by the TR-32052 project of the Serbian Ministry of Education, Science and Technological Development. The authors would like to thank prof. Alina Caddemi, University of Messina, Italy, for providing the measured data.

\section{REFERENCES}

[1] R. A. Pucel, H. A. Haus, H. Statz, "Signal and noise properties of gallium arsenide microwave fieldeffect transistors," Advances in Electronics and Electron Physics, vol. 38, pp. 195-265, 1975. [Online]. Available: http://www.sciencedirect.com/science/article/pii/S0065253908612056

[2] R. P. Meys, "A wave approach to the noise properties of linear microwave devices," IEEE Transactions on Microwave Theory and Techniques, vol. 26, no. 1, pp. 34-37, 1978. [Online]. Available: http://ieeexplore.ieee.org/document/1129303/

[3] H. Fukui, "Design of microwave GaAs MESFET's for broad-band low-noise amplifiers," IEEE Transactions on Microwave Theory and Techniques, vol. 27, no. 7, pp. 643-650, 1979. [Online]. Available: http://ieeexplore.iee.org/abstract/document/1129694/

[4] A. Cappy, A. Vanoverschelde, A. Schortgen, C. Versnaeyen, G. Salmer, "Noise modeling in submicrometer-gate two-dimensional electron-gas field-effect transistors," IEEE Transactions on Electron Devices, vol. 32, no. 12, pp. 2787-2795, 1985. [Online]. Available: http://ieeexplore.ieee.org/document/1485163/

[5] M. S. Gupta, O. Pitzalis, S. E. Rosenbaum, P. T. Greiling, "Microwave noise characterization of GaAs MESFETs: evaluation by on-wafer low-frequency output noise current measurement," IEEE Transactions on Microwave Theory and Techniques, vol. 35, no. 12, pp. 1208-1218, 1987. [Online]. Available: http://ieeexplore.ieee.org/document/1133839/

[6] M. W. Pospieszalski, "Modeling of noise parameters of MESFET's and MODFET's and their frequency and temperature dependence," IEEE Transactions on Microwave Theory and Techniques, vol. 37, no. 9, pp. 1340-1350, 1989. [Online]. Available: http://ieeexplore.ieee.org/document/32217/ 
[7] S. W. Wedge, D. B. Rutledge, "Wave techniques for noise modeling and measurement," IEEE Transactions on Microwave Theory and Techniques, vol. 40, no. 11, pp. 2004-2012, 1992. [Online]. Available: http://ieeexplore.ieee.org/document/168757/

[8] O. Pronić, V. Marković, N. Maleš-Ilić, "The wave approach to noise modeling of microwave transistors by including the correlation effect," Microwave and Optical Technology Letters, vol. 28, no. 6, pp. 426-430, 2001. [Online]. Available: http://onlinelibrary.wiley.com/doi/10.1002/10982760(20010320)28:6\%3C426::AID-MOP1061\%3E3.0.CO;2-J/abstract

[9] O. Pronić, V. Marković, "A wave approach to signal and noise modeling of dual-gate MESFET," AEU International Journal of Electronics and Communications, vol. 56, no. 1, pp. 61-64, 2002. [Online]. Available: http://www.sciencedirect.com/science/article/pii/S1434841104700643

[10] R. P. Hecken, "Analysis of liner noisy two-ports using scattering waves," IEEE Transactions on Microwave Theory and Techniques, vol. 29, no. 10, pp. 997-1004, 1981. [Online]. Available: http://ieeexplore.ieee.org/document/1130490/

[11] J. A. Dobrowolski, Computer-aided analysis, modeling and design of microwave networks-the wave approach. Artech House, Norwood, 1996.

[12] O. Pronić, V. Marković, N. Maleš-Ilić, "MESFET noise modeling based on noise wave temperatures," in Proceedings of 4th International Conference on Telecommunications in Modern Satellite, Cable and Broadcasting Services, TELSIKS 1999, Niš, Yugoslavia, pp. 407-410, 1999. [Online]. Available: http://ieeexplore.iee.org/document/806241/

[13] O. Pronić-Rančić, V. Marković, "Microwave transistors noise modeling by using variable noise wave temperatures", in Proceedings of 5th International Conference on Telecommunications in Modern Satellite, Cable and Broadcasting Services, TELSIKS 2001, Niš, Yugoslavia, pp. 313-316, 2001. [Online]. Available: http://ieeexplore.ieee.org/document/954899/

[14] V. Marković, O. Pronić-Rančić, Z. Marinković, "Noise wave modeling of microwave transistors based on neural networks," Microwave and Optical Technology Letters, vol. 41, no. 4, pp. 294-297, 2004. [Online]. Available: http://onlinelibrary.wiley.com/doi/10.1002/mop.20120/abstract?wol1URL=/doi/ 10.1002/mop.20120/abstract\&regionCode=RS\&identityKey=f98a7459-911e-4243-8566-5461bca08a4b

[15] D. Pasquet, E. Bourdel, S. Quintanel, T. Ravalet, P. Houssin, "New method for noise-parameter measurement of a mismatched linear two-port using noise power wave formalism," IEEE Transactions on Microwave Theory and Techniques, vol. 56, no. 9, pp. 2136-2142, 2008. [Online]. Available: http://ieeexplore.ieee.org/document/4599243/

[16] J. A. Dobrowolski, Microwave network design using the scattering matrix. Artech House, Norwood, 2010.

[17] J. A. Dobrowolski, "Noise characterization of differential multi-element multiport networks - the wave approach," International Journal of Electronics and Telecommunications, vol. 61, no. 4, pp. 395-401, 2015. [Online]. Available: https://www.degruyter.com/view/j/eletel.2015.61.issue-4/eletel-20150052/eletel-2015-0052.xml

[18] V. Đorđević, Z. Marinković, G. Crupi, O. Pronić-Rančić, V. Marković, A. Caddemi, "Wave approach for noise modeling of gallium nitride high electron-mobility transistors," International Journal of Numerical Modelling: Electronic Networks, Devices and Fields, 2015. [Online]. Available: http://onlinelibrary.wiley.com/doi/10.1002/jnm.2138/abstract

[19] G. Crupi, D. M.M.-P. Schreurs, Microwave de-embedding from theory to applications, Academic Press, Oxford, 2013.

[20] A. Nalli, A. Raffo, G. Crupi, S. D’Angelo, D. Resca, F. Scappaviva, G. Salvo, A. Caddemi, G. Vannini, "GaN HEMT noise model based on electromagnetic simulations," IEEE Transactions on Microwave Theory and Techniques, vol. 63, no. 8, pp. 2498-2508, 2015. [Online]. Available: http://ieeexplore.ieee.org/abstract/ document/7153571/

[21] V. Đorđević, Z. Marinković, O. Pronić-Rančić, V. Marković, "Analytical Procedure for Extraction of HEMT Noise Wave Temperatures", in Proceedings of the 3rd International Conference on Electrical, Electronic and Computing Engineering, IcETRAN 2016, Zlatibor, Serbia, pp. MTI 2.6 1-6, 2016.

[22] P. K. Ikalainen, "Extraction of device noise sources from measured data using circuit simulator software", IEEE Transactions on Microwave Theory and Techniques, vol. 41, no. 2, pp. 340-343, 1993. [Online]. Available: http://ieeexplore.ieee.org/document/216478/

[23] V. Đorđević, Z. Marinković, O. Pronić-Rančić, V. Marković, "Extraction of intrinsic noise parameters of microwave FETs based on ANN", in Proceedings of the 1st International Conference on Electrical, Electronic and Computing Engineering, IcETRAN 2014, Vrnjačka Banja, Serbia, pp. MTI 2.2 1-5, 2014.

[24] A. Caddemi, A. Di Paola, M. Sannino, "Microwave noise parameters of HEMTs vs. temperature by a simplified measurement procedure," in Proceedings of High Performance Electron Devices for 
Microwave and Optoelectronic Applications Workshop, EDMO 1996, Leeds, UK, pp. 153-157, 1996. [Online]. Available: http://ieeexplore.ieee.org/document/575819/

[25] H. Hillbrand, P. Russer, "An efficient method for computer aided noise analysis of linear amplifier networks," IEEE Transactions on Circuits and Systems, vol. cas-23, no. 4, pp. 235-238, 1976. [Online]. Available: http://ieeexplore.ieee.org/document/1084200/?section=abstract

[26] J. E. Rayas-Sanchez, "EM-based optimization of microwave circuits using artificial neural networks: The state-of-the-art", IEEE Transactions on Microwave Theory and Techniques, vol. 52, no. 1, pp. 420-435, 2004. [Online]. Available: http://ieeexplore.ieee.org/document/1262733/

[27] Z. Marinković, O. Pronić-Rančić, V. Marković, "ANN applications in improved noise wave modeling of microwave FETs", Microwave and Optical Technology Letters, vol. 50, no. 10, pp. 2512-2516, 2008. [Online]. Available: http://onlinelibrary.wiley.com/doi/10.1002/mop.23771/abstract

[28] H. Kabir, L. Zhang, M. Yu, P. Aaen, J. Wood, Q. J. Zhang, "Smart modeling of microwave devices", IEEE Microwave Magazine, vol. 11, no. 3, pp. 105-108, 2010. [Online]. Available: http://ieeexplore.ieee. org/document/5450640/

[29] Z. Marinković, N. Ivković, O. Pronić-Rančić, V. Marković, A. Caddemi, "Analysis and validation of neural approach for extraction of small-signal models of microwave transistors", Microelectronics Reliability, vol. 53, no. 3, pp. 414-419, 2013. [Online]. Available: http://www.sciencedirect.com/ science/article/pii/S0026271412004179

[30] E. Petrović, Ž. Ćojbašić, D. Ristić-Durrant, V. Nikolić, I. Ćirić, S. Matić, "Kalman filter and narx neural network for robot vision based human tracking", Facta Universitatis, Series: Automatic Control and Robotics, vol. 12, no. 1, pp. 43-51, 2013. [Online]. Available: http://casopisi.junis.ni.ac.rs/index.php/ FUAutContRob/article/view/17

[31] M. Milovanović, D. Antić, S. Nikolić, M. Spasić, S. Perić, M. Milojković, "Data denoising procedure for neural network performance improvement", Facta Universitatis, Series: Automatic Control and Robotics, vol. 14, no. 3, pp. 173-182, 2015. [Online]. Available: http://casopisi.junis.ni.ac.rs/index.php/ FUAutContRob/article/view/1370

[32] I. Pavlović, I. Ćirić, R. Pavlović, M. Simonović, V. Nikolić, "Viscoelastic double beam system stability analysis using artificial neural networks", Facta Universitatis, Series: Automatic Control and Robotics, vol. 14, no. 1, pp. 11-17, 2015. [Online]. Available: http://casopisi.junis.ni.ac.rs/index.php/ FUAutContRob/article/view/784

[33] Z. Marinković, V. Marković, T. Ćirić, L. Vietzorreck, O. Pronić-Rančić, "Artificial neural networks in RF MEMS switch modelling", Facta Universitatis, Series: Electronics and Energetics, vol. 29, no. 2, pp. 177-191, 2016. [Online]. Available: http://casopisi.junis.ni.ac.rs/index.php/FUElectEnerg/article /view/1293

[34] B. Predić, M. Mandić, M. Roganović, M. Kovačević, D. Stojanović, "Prediction of passenger car fuel consumption using artificial neural network: a case study in the city of Niš", Facta Universitatis, Series: Automatic Control and Robotics, vol. 15, no. 2, pp. 105-116, 2016. [Online]. Available: http://casopisi.junis.ni.ac.rs/index.php/FUAutContRob/article/view/1597

[35] M. Simonović, V. Nikolić, E. Petrović, "Input vector impact on short-term heat load prediction of small district heating system", Facta Universitatis, Series: Automatic Control and Robotics, vol. 15, no. 2, pp. 95-103, 2016. [Online]. Available: http://casopisi.junis.ni.ac.rs/index.php/FUAutContRob/article/ view/1724

[36] Q. J. Zhang, K. C. Gupta, Neural networks for RF and microwave design. Artech House, Boston, 2000.

[37] MATLAB, The Language of Technical Computing, 2012. [Online]. Available: http://www.mathworks. com/products/matlab

[38] ADS, Advanced Design System, 2002. [Online]. Available: http://www.keysight.com/en/pc1297113/advanced-design-system-ads?cc=RS\&lc=eng

[39] ADS, Advanced Design System, 2009. [Online]. Available: http://www.keysight.com/en/pc1297113/advanced-design-system-ads?cc=RS\&lc=eng 